EPJ Web of Conferences 81, 03009 (2014)

DOI: $10.1051 /$ epjconf/ 20148103009

(C) Owned by the authors, published by EDP Sciences, 2014

\title{
Search for a new light boson in $\eta$ decays using the WASA detector
}

\author{
Joanna Stepaniak ${ }^{1, a}$ and Marcin Berlowski ${ }^{1}$ \\ for the WASA-at-COSY Collaboration \\ ${ }^{1}$ National Centre for Nuclear Research, Warsaw, Poland
}

\begin{abstract}
Some dark matter scenarios predict a light scalar boson that couples to charged leptons. Present experiments do not exclude the existence of a new light $(\mathrm{M}<1 \mathrm{GeV})$ particle(s) with small coupling to leptons. It can manifest itself in $\eta$ meson decays with electron-positron pair(s)in the final state. We present preliminary results from the search for $\eta \rightarrow \pi^{0}+\left(X \rightarrow e^{+} e^{-}\right)$decay in a total of $5 \times 10^{7} \eta$ mesons produced in the $p p \rightarrow$ $p p \eta$ reaction, a fraction of the data collected in the WASA experiment.
\end{abstract}

\section{Introduction}

The possibility of the existence of a very weakly coupled light boson mediating dark matter interactions has received much attention recently. Searches for such vector boson were performed among others in meson decays $[1,2]$. It is possible that the hidden sector has a richer structure, therefore one can also consider a signal from a new scalar particle. The $\eta \rightarrow \pi^{0} e^{+} e^{-}$decay is a good place to search for a weakly interacting scalar boson coupling to leptons because $\mathrm{C}$-parity forbids this decay to occur as a single photon SM process. The decay rate via the two-photon intermediate state was calculated and the expected value was found to be of the order of $10^{-8}[3,4]$. The decay has not yet been observed and an experimental upper limit of the branching ratio of $4 \times 10^{-5}$ exists [5]. Theoretical upper bound for $\eta \rightarrow \pi^{0}\left(X \rightarrow e^{+} e^{-}\right)$branching fraction where $\mathrm{X}$ is a new light hypothetical scalar boson was predicted by Kozlov [6] to be of the order of $10^{-5}$. Leutwyler and Shifman [7] expected the value about $p_{\pi} / m_{\eta} \times 10^{-6}$ only weakly depending on the boson mass.

A large number of $\eta$ meson decays were collected with WASA in several experiments. Both proton-proton reaction at $1.4 \mathrm{GeV}$ and pd reactions at $1 \mathrm{GeV}$ incident proton energy was used as a source of the $\eta$ mesons. We present here preliminary results based on a fraction of available statistic collected with a specific trigger, which original was the search for very rare $\eta \rightarrow e^{+} e^{-}$decay. From that search the upper limit for the Branching Ratio $3.9 \times 10^{-6}$ was extracted with $90 \%$ confidence level Ref. [8].

\section{The Experiment}

The experiment was performed with the WASA detector setup at the COSY accelerator (see Fig. 1a). The WASA facility allows the simultaneous measurement of charged and neutral particles that is crucial for $\eta \rightarrow \pi^{0} e^{+} e^{-}$and $\eta \rightarrow e^{+} e^{-} \gamma$ decay channels. The $\eta$ mesons were produced in $p p \rightarrow$

\footnotetext{
ae-mail: Joanna.Stepaniak@ fuw.edu.pl
} 
(a)

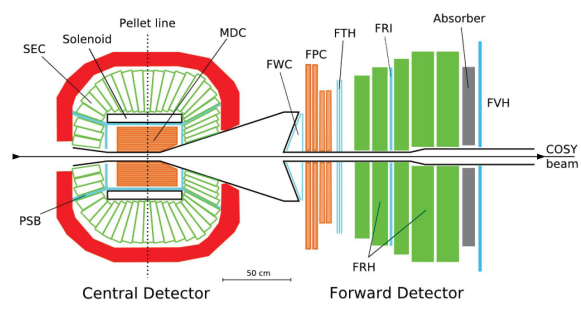

(b)

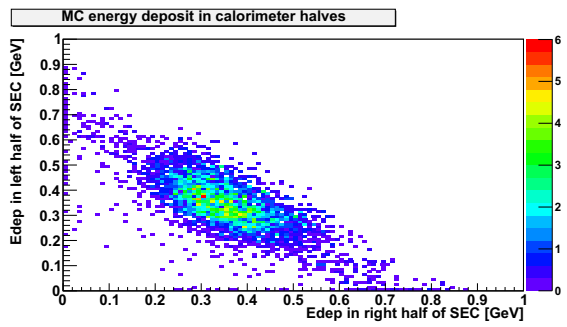

Figure 1. (a) Schematic side view of the WASA detector, (b) Distribution of energy deposits in the left and the right halves of the calorimeter for the simulated $\eta \rightarrow e^{+} e^{-} \gamma$ decay after the reconstruction in the detector.

$p p \eta$ interactions at $1.4 \mathrm{GeV}$ incident proton energy. At the energy considered being not far from the threshold for the $\eta$ meson production both protons are emitted at small angles with respect to the beam particles. Therefore the observation of two charge tracks in the forward part of the apparatus were used for the $\eta$ tagging and requested by the trigger. The Forward Detector covers the angular range 3-18 degrees with respect to the beam direction. The minimum energy of each forward track was required to be larger than $50 \mathrm{MeV}$. The decay products of the $\eta$ meson are preferably emitted at large angles and were detected in the central part of the Electromagnetic Calorimeter and in the Drift Chamber. The data presented here was collected with a trigger that required in addition a large energy deposit in the two, left and right half of the central part of Electromagnetic Calorimeter. The Fig. 1b shows the plots of simulated energy deposits in the left and the right half of the calorimeter originated from the $\eta \rightarrow e^{+} e^{-} \gamma$ decays. One can observe that both energies are predominantly large therefore the trigger acceptance was satisfactory.

\section{The $\eta \rightarrow e^{+} e^{-} \gamma$ decay channel}

The Dalitz eta decay $\eta \rightarrow e^{+} e^{-} \gamma$ was studied to check the quality of the electron reconstruction and identification in the detector as well as to check the normalization assuming the branching fraction known from the theory and from the experiment. Moreover this channel is a main source of background to $\eta \rightarrow \pi^{0} e^{+} e^{-}$decay in the region of small $e^{+} e^{-}$invariant mass. The region of low $e^{+} e^{-}$ masses was explored to avoid the influence of the not so well known transition form factor of the $\eta$ meson. Therefore, at first step of the selection the $\eta \rightarrow e^{+} e^{-} \gamma$ decay candidates from the collected data the class of events with one photon of energy larger than $20 \mathrm{MeV}$ and two charged tracks in CD with $M_{e e}$ less than $125 \mathrm{MeV}$ was selected. The cut on $20 \mathrm{MeV}$ has been justified by MC simulations. The invariant mass was calculated assuming the electron masses for both charged particles. For such events the two proton missing mass as a function of $e^{+} e^{-} \gamma$ invariant mass $\left(M_{e e \gamma}\right)$ is plotted in Fig. 2. The accumulation of events with both two proton missing $\operatorname{mass}\left(M M_{p p}\right)$ and $e^{+} e^{-} \gamma$ invariant mass $\left(M_{e e \gamma}\right)$ around the eta mass is clearly seen. After cuts on $M M_{p p}$ and $M_{e e \gamma}$ we still observe some admixture of events from $\eta \rightarrow e^{+} e^{-} \gamma$ decays with the $\gamma$ conversion mainly in the thin cylindrical beryllium beam tube of a $30 \mathrm{~mm}$ radius and thickness $1.2 \mathrm{~mm}$. The conversion probability in the 30 $\mu m$ windowless hydrogen target can obviously be neglected. The elimination of the external conversion of photons was based on the rejection of events with very small $M_{e e}$ calculated from momenta at the beam tube. After the subtraction of such events the $M_{e e}$ distribution satisfactorily agreed with the simulated distribution of $e^{+} e^{-} \gamma$ decay reconstructed events [8]. The comparison of the charged track 


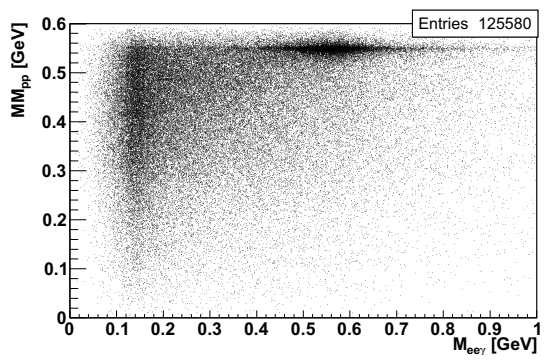

Figure 2. Distribution of the missing mass of two tagging protons as a function of the $e^{+} e^{-} \gamma$ invariant mass for the events with two charged tracks, one neutral shower and $M_{e e}<0.125 \mathrm{GeV}$.

momentum measured in the Drift Chamber with the energy released in the Electromagnetic Calorimeter was used for the electron identification. The extracted branching fraction for $\eta \rightarrow e^{+} e^{-} \gamma$ decay was found to be consistent within errors with the current world average.

\section{Search for $\eta \rightarrow \pi^{0} e^{+} e^{-}$decay}

A sample of event candidates for $\eta \rightarrow \pi^{0} e^{+} e^{-}$decay were selected with the requirements applied in three steps: (a) events with two charged particles with opposite charge, two neutral clusters in the Electromagnetic Calorimeter $(\mathrm{E}>10 \mathrm{MeV})$, at least two tracks in the Forward Detector and the two-proton missing mass in the interval (0.5-0.6) GeV were selected, (b) the rejection of external conversion of photons was performed using the procedure described in Ref. [8] and the cut on the two photon invariant mass around the $\pi^{0}$ mass $\left(0.11<M_{\gamma \gamma}<0.165 \mathrm{GeV}\right)$ was applied, (c) the cut on the ratio of momentum measured in the Drift Chamber to energy deposited in calorimeter $p / E_{d e p}<1.2$ for each charged track was used to remove an admixture of pions. Finally the more stringent cut on $M M_{p p}$ and on the deficit of the total observed energy was applied to diminish the admixture of direct two and three pion production. The distribution of two charged tracks invariant mass is presented in Figs. $3 \mathrm{a}, \mathrm{b}, \mathrm{c}$. The second maximum in the figure (a) can be explained by a photon conversion in the $1.2 \mathrm{~mm}$ thick Beryllium beam tube situated at the $30 \mathrm{~cm}$ from the primary vertex since the dilepton mass was calculated using momenta extrapolated to the assumed target position. After the rejection of the conversion and keeping only events with two photon invariant mass in the $\pi^{0}$ mass region 1623 events were retained in the sample of candidates (see Fig. 3b). A flat maximum around $300 \mathrm{MeV}$ was found to be consistent with an admixture of $\eta$ decays containing a charged pion pair. An example of the distribution for the $\eta \rightarrow \pi^{+} \pi^{-} \pi^{\circ}$ is shown in Fig. 3 d. After the final selection cut (Fig. 3c) no events were observed with $M_{e e}$ larger than $100 \mathrm{MeV}$. Several background channels were considered and simulated : (i) the $\eta \rightarrow e^{+} e^{-} \gamma, \eta \rightarrow \gamma \gamma, \eta \rightarrow \pi^{+} \pi^{-}\left(\pi^{\circ} \rightarrow \gamma \gamma\right), \eta \rightarrow \pi^{+} \pi^{-}\left(\pi^{\circ} \rightarrow e^{+} e^{-} \gamma\right)$ and $\eta \rightarrow \pi^{+} \pi^{-} \gamma$ decays (ii) the direct pion production reactions $p p \rightarrow p p\left(2 \pi^{\circ} \rightarrow \gamma \gamma\right)\left(\pi^{\circ} \rightarrow e^{+} e^{-} \gamma\right)$ and $p p \rightarrow p p \pi^{+} \pi^{-}\left(\pi^{\circ} \rightarrow \gamma \gamma\right)$. After the last selection only $\eta \rightarrow e^{+} e^{-} \gamma$ decay admixture was found to be on the level compatible with the candidate observed in the low mass region and expected amount of such events explained the number of the events observed in the low mass region. The direct production of pions as well as $\eta \rightarrow \gamma \gamma$ decay admixture appeared to be negligible.

\section{Summary}

Search for the rare decay $\eta \rightarrow \pi^{0} e^{+} e^{-}$in a sample of about 4 million of reconstructed $\eta$ decays was performed and no signal of such decay with invariant mass of $e^{+} e^{-}$pair in the interval $100-450 \mathrm{MeV}$ 
(a)

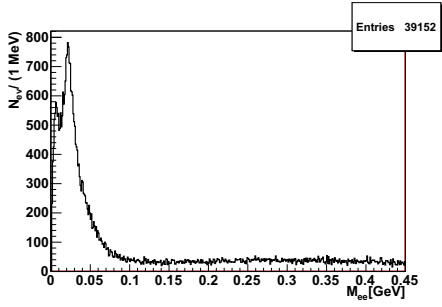

(c)

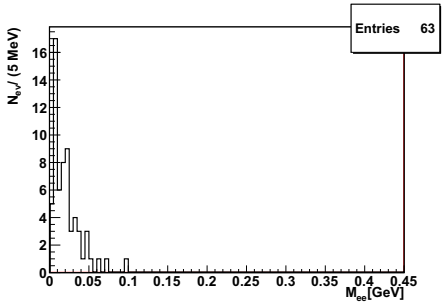

(b)

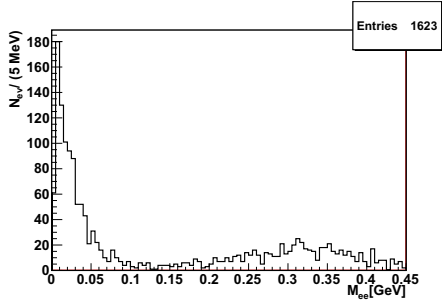

(d)



Figure 3. Distributions of the lepton pair mass after subsequent rejections a, b, c. The shape of the $M_{e e}$ distribution for the $\eta \rightarrow \pi^{+} \pi^{-} \pi^{0}$ with electron masses assumed for the charged pions (d). The distribution presented in (d) corresponds to the same cut as used for step (b).

was observed. It corresponds to a preliminary upper limit of $2.0 \times 10^{-5}$ for the BR of the decay into $\pi^{0}+\left(X \rightarrow e^{+} e^{-}\right)$. As a cross check we measured the $\eta \rightarrow e^{+} e^{-} \gamma$ branching fraction and found it to be consistent with the current world average. A search for C-violating process with one virtual pion was already performed using WASA detector in the PhD Thesis Ref. [9, 10]. For such process the enhancement at small $M_{e e}$ is expected. Therefore the low mass region was explored and a limit of $3.7 \times 10^{-5}$ was given for $M_{e e}<40 \mathrm{MeV}$. The WASA Collaboration collected already an order of magnitude larger statistics of eta decays which may permit to achieve the sensitivity requested by theoretical expectation for the new hypothetical boson. Data analysis is in progress for the full data set.

\section{Acknowledgements}

Supported by the Polish National Science Center under grants No. 0320/B/H03/2011/40, 2011/03/B/ST2/01847.

\section{References}

[1] R. Essig, J.A. Jaros, W. Wester, arXiv:1311.0029 [hep-ph] (2013) and references therein.

[2] P. Adlarson et al. /WASA-at-COSY Collaboration, Phys.Lett. B 726 187-193 (2013)

[3] T.P. Cheng, Phys.ReV.162, 1734 (1967)

[4] J.N. Ng and D.J. Peters, Phys.Rev. D 46, 5034-5039 (1992)

[5] K.A. Olive(Particle Data Group), Chin.Phys. C 38, 090001 (2014)

[6] G.A. Kozlov, Chin.J.Phys. 34920 (1996), Nuovo Cimento A 109, 207 (1996)

[7] A. Leutwyler and M.A. Shifman, Nucl.Phys. B 343, 369 (1990)

[8] M. Berlowski, PhD Thesis, National Centre for Nuclear Research, Warsaw, Poland (2013)

[9] A. Winnemöller, PhD Thesis, Universität Münster, Germany (2011) (in German)

[10] M. Zielinski, PhD Thesis, Jagiellonian University, Cracow, Poland (2012), arXiv:1301.0098 